\title{
Pseudarthrosen am Sakrum
}

Ulf Culemann

\section{Einleitung}

Eine Pseudarthrose ist definiert als Knochenheilungsstörung, die nicht zu einer knöchernen Ausheilung der Frakturenden geführt hat, sondern lediglich bindegewebig heilt. Eine mechanische Belastbarkeit der Kraftübertragung im hinteren Beckenring beim Stehen, Sitzen und Gehen ist mit einer Pseudarthrose im Sakrumbereich daher nicht zu erzielen. Der Patient verspürt hierbei zunehmende Schmerzen tief lumbal, er hat Schmerzen „im Kreuz“.

Normalerweise sind dorsale Beckenringfrakturen oder Sakrumfrakturen nach 3-4 Monaten knöchern fest verheilt. Besteht nach 6 Monaten keine eindeutige radiologisch nachweisbare Durchbauung, ist die Fraktur aber klinisch fest und der Patient gibt keinerlei Schmerz an, spricht man am Becken noch von einer verzögerten Frakturheilung. Lässt sich nach 6-12 Monaten immer noch keine Durchbauung der Sakrumfraktur am Beckenring in der CT nachweisen und bestehen entsprechende klinische Schmerzen in der Frakturregion am Patienten, liegt definitionsgemäß eine Pseudarthrose vor [1]. Ursachen für die Ausbildung einer Pseudarthrose sind bei Sakrumfrakturen wie auch in anderen anatomischen Regionen in 3 Kategorien zu unterteilen: mechanische, biologische oder infektionsbedingte Heilungsstörungen des hinteren Beckenrings. Die Fehlverheilungen am hinteren Beckenring lassen sich nach Mears und Velyvis in 4 Typen unterschieden [2]:

- Nichtheilung ohne Fehlstellung, die eigentliche „Nonunion“ ( $\triangleright$ Abb. 1)

- verknöcherte Fehlstellung („united malalignment“), die eigentliche „Malunion“

- nicht verknöcherte Fehlstellung („ununited malalignment";

- partiell verknöcherte Fehlstellung („partially united malalignment";

Am Sakrum tritt zumeist eine Nichtheilung ohne Fehlstellung auf.

\section{Epidemiologie und Ursachen von Pseudarthrosen am Sakrum}

Im vorderen Beckenringbereich finden sich nach Schambeinastfrakturen häufiger pseudarthrotische Fehlverheilungen als im hinteren Beckenringbereich. Aufgrund der biomechanischen Stabilität einer Typ-A-Verletzung des Beckens in der Klassifikation der AO werden diese Frakturtypen i.d. R. rein konservativ therapiert [3]. Bedingt durch eine anhaltende Mikrobewegung der „Stoßstangen des Beckenrings“ (= Schambeinäste) beim Gehen, können diese Frakturen gelegentlich nur sehr verzögert oder eben tatsächlich nicht verheilen. Radiologisch beschriebene Pseudarthrosen am vorderen Beckenring ohne entsprechende klinische Symptome, wie z. B. Schmerzen in Ruhe nach direktem Druck oder auftretende Schmerzen bei Bewegung, sind keine Pseudarthrosen, sondern es besteht bildmorphologisch keine Durchbauung. Da sie dem Patienten aber keine Schwierigkeiten bereiten, sind dies nur röntgenbildmorphologisch fehlende Durchbauungszeichen. Sie haben für den Patienten klinisch keine Relevanz.

„Echte“ Pseudarthrosen sind bildmorphologisch eindeutig nachweisbar und machen dem Patienten klinisch dann zumeist auch starke Schmerzen. Diese können sowohl in Ruhe auf Druck oder unter der Gehbelastung auftreten und weisen häufig bei gezielter Nachfrage einen eher zunehmenden Charakter im Verlauf auf.

Pseudarthrosen im hinteren Beckenring und hier speziell im Sakrumbereich verhalten sich demgegenüber anders. Sakrumpseudarthrosen sind nicht nur sehr selten, sondern die fehlende knöcherne Durchbauung im Sakrum führt in hohem Maße sofort zu Schmerzen und zu einer Gang- und Sitzunfähigkeit, wenn sie auftreten [4]. Grundsätzlich sind Beckenringfrakturen mit Instabilität des hinteren Beckenrings (Typ B+C nach AO) Frakturen, die sich sowohl durch eine translatorische (vertikal) als auch durch eine rotatorische (sagittal) Instabilität auszeichnen und häufig zu einer Dislokation im Frakturbereich führen $[3,5,6,7]$. Sie müssen aufgrund der biomechanischen Instabilität adäquat operativ versorgt werden. Unterbleibt die operative Stabilisierung der Sakrumfraktur aufgrund einer übersehenen Fraktur oder nicht erkannten Instabilität, kann eine Pseudarthrose auftreten. Im Sakrum be- 

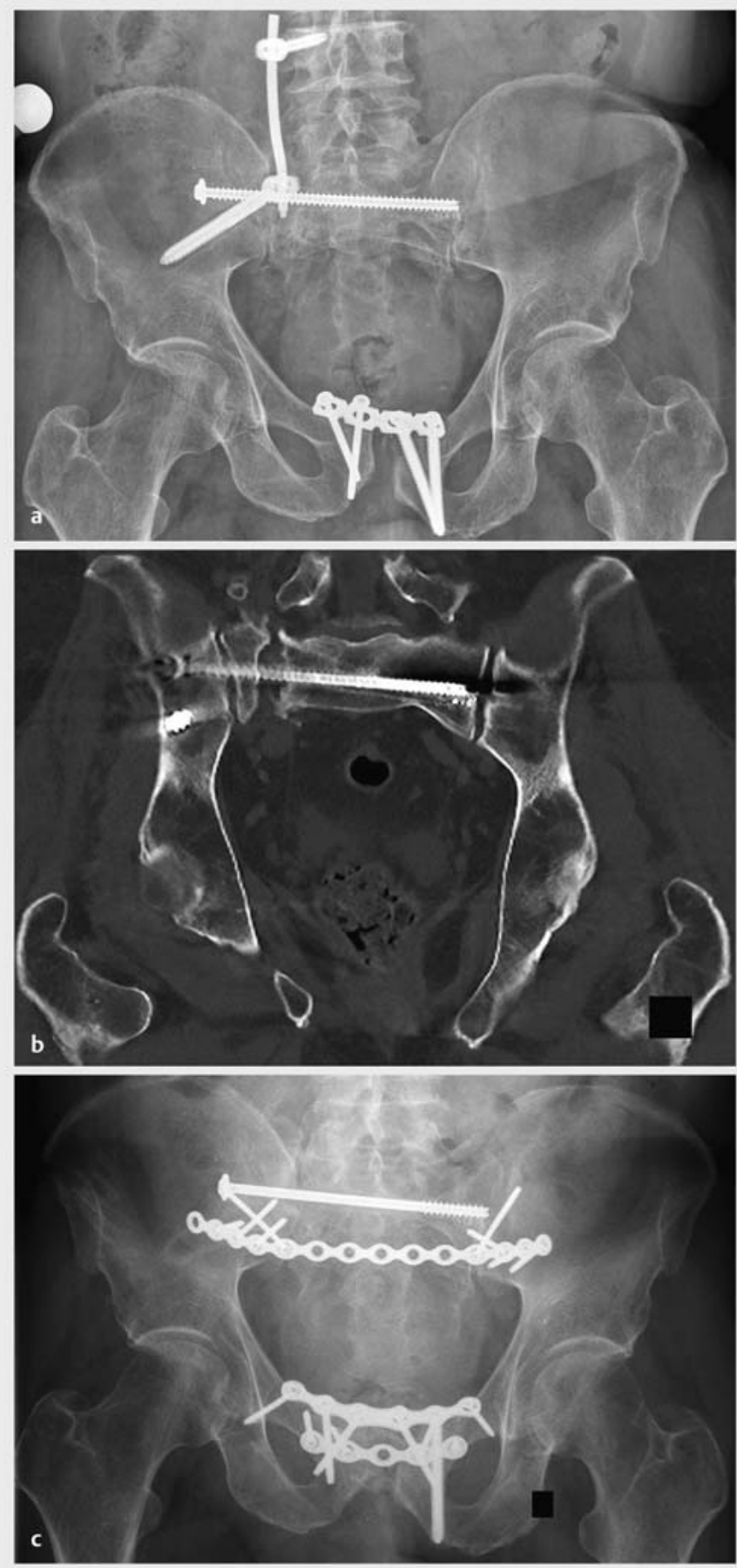

- Abb. 1 Nonunion einer mehrfragmentären Sakrumfraktur rechts mit Schraubenlockerungen nach triangulärer lumbopelviner Stabilisierung und Symphysenplatte bei Symphysenruptur im Rahmen einer vertikal instabilen Verletzung. Beckenübersicht (a) und koronares CT-Schnittbild mit Defektzone in der Massa lateralis, Sakrum rechts und gelockerten L-IV-Pedikel- und Iliumschrauben (b) vor Pseudarthrosenrevision. Beckenübersicht 2 Monate nach Symphysiodese mit Doppelplatten und Beckenspan sowie dorsaler Entfernung der Instrumentierung, Beckenspaninterposition sakral, neuer iliosakraler Schraube und dorsaler Zuggurtungsplatte (c). Quelle: Keel MJB, Bastian JD. Spätfolgen - Beckenchirurgie. OP-Journal 2018; 34: 48-52. trifft dies nach der Einteilung von Denis et al. [27] häufig Frakturen, die in der Zone 2 und 3, also in der transforaminalen und oder zentralen Frakturzone am Sakrum auftreten. Aufgrund der biomechanisch notwendigen Kraftübertragung von der Wirbelsäule auf beide Beine, die im hinteren Becken durch das Sakrum an beiden Seiten stattfindet, führt eine bleibende mechanische Instabilität unter fortdauernder Belastung zur Pseudarthrose und somit zu entsprechenden Schmerzen [6, 28, 10, 11-16].

Durch die äußerst straffen Bandverbindungen der unteren Wirbelsäule mit dem Ilium (iliosakrale Bänder, Beckenbodenbänder sakrospinal und sakrotuberal) ist das Sakrum selbst im Verletzungsfalle noch gut im hinteren Beckenring fixiert und ausreichend stabil „verpackt“ [7, 17]. Frakturen in den Sakrumflügeln (Ala sacralis) stellen aufgrund der guten spongiösen Knochenstruktur und Durchblutung des Sakrums daher selten eine Ursache für die Ausbildung einer Pseudarthrose dar. Transalare Sakrumfrakturen (=Zone 1 nach Denis et al. [27]) werden beim jungen Patienten zusätzlich durch die elastischen Bandstrukturen ausreichend geschützt, sodass sie trotz der bestehenden Rotationsinstabilität des Beckens (Typ-B-Verletzung nach AO) ausheilen können. Aufgrund der zunehmenden Verkalkung des hinteren Bandapparates (Ligg. sacroiliacalia dorsalia) und der daraus resultierenden zunehmenden Steifigkeit des Beckenringes dorsal beim alten Patienten, sind die transalaren Frakturtypen als sog. Insuffizienzfrakturen des Beckens (Typ C+D der FFP-Klassifikation nach Rommens et al. [18]) deutlich häufiger von einer Pseudarthrosenentstehung betroffen. Insbesondere bei hochaltrigen Patienten kommt durch die zusätzlich bestehende Rarefizierung des spongiösen Knochens in der Ala sacralis hinzu, dass trotz operativ korrekt ausgeführter Stabilisierung eine fortgesetzte Instabilität mit Osteosyntheselockerung verbleibt und hieraus häufiger ebenfalls eine Pseudarthrose resultieren kann [18].

Bei transforaminalen Frakturtypen kann es durch die kleine Kontaktfläche im foraminalen Frakturbereich ohne ausreichende Verzahnung oder auch durch biologische Ursachen (z.B. durch eine höhergradige Weichteilschädigung bei Granatsplitterverletzungen oder eine lokale Infektion bei Dekubitalulzera beim bettlägerigen Patienten) ebenfalls häufiger zu einer Pseudarthrose im Sakrumbereich kommen.

Am weitaus häufigsten entstehen Sakrumpseudarthrosen jedoch durch schlichtes „Übersehen“ der Fraktur ( Abb. 2).

Die fortgesetzten Mikrobewegungen in der Sakrumfraktur bei Belastung im Stehen, Sitzen und Gehen (fehlende mechanische Stabilität) führen daher zu anhaltenden Beschwerden und Entwicklung der Pseudarthrose, ebenfalls 

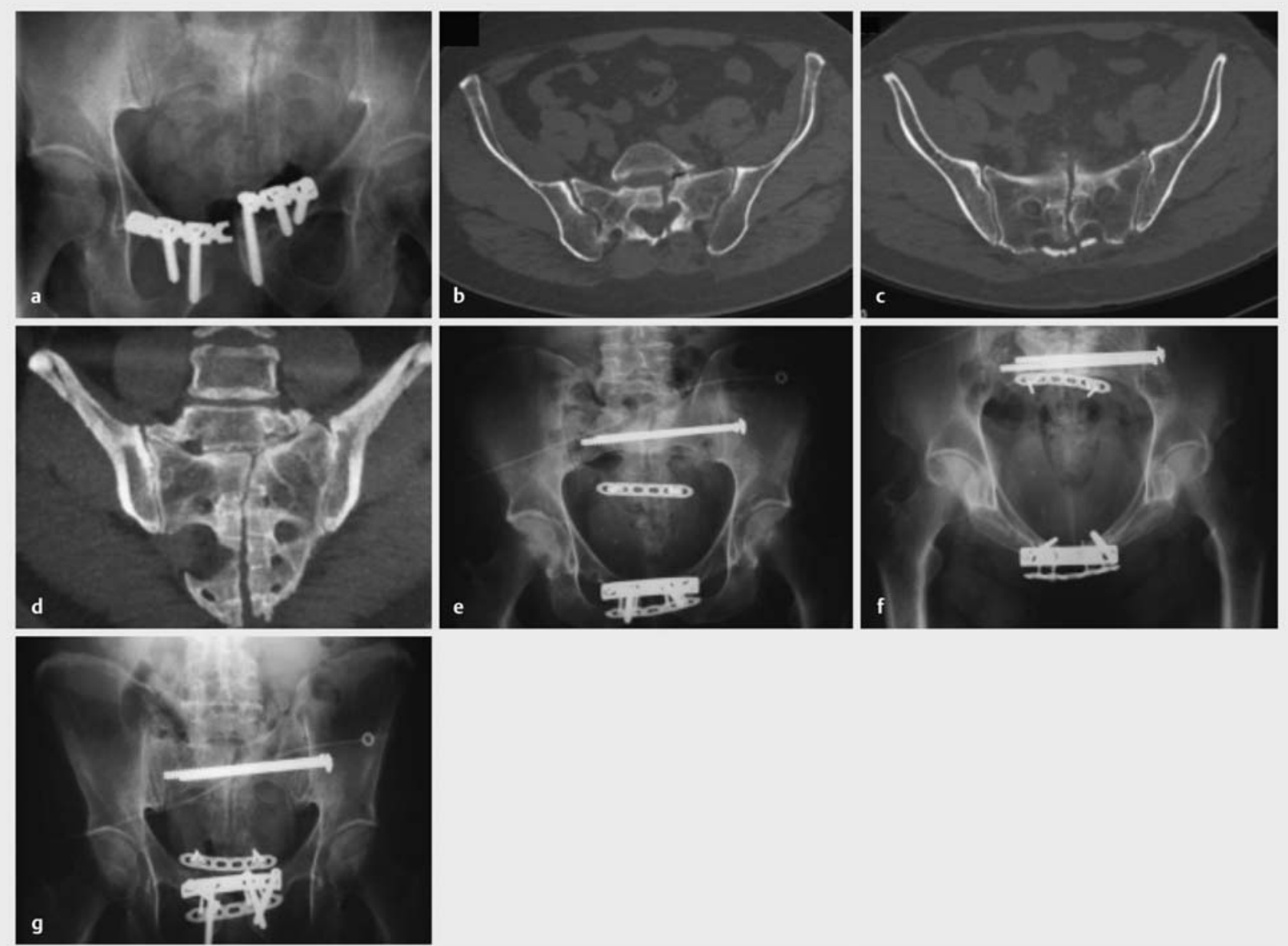

- Abb. 2 Darstellung einer übersehenen hinteren Beckenringfraktur, die primär lediglich ventral stabilisiert wurde. Nach Mobilisation Plattenbruch ( $\vee$ Abb. 3a). Die Unfall-CT zeigt eine Längs- und Querfraktur des Sakrums ( $\bullet$ Abb. 3b-d), die einer Berstungsfraktur des Sakrums entspricht und damit instabil ist. Dorsale Stabilisierung mit SI-Schraubenosteosynthese und ventraler Reosteosynthese durch Doppelplatten $(\triangleright \mathbf{A b b}$. $\mathbf{3 e - g}$ ). Quelle: Culemann U, Tosounidis G, Pohlemann T. Lösungsmöglichkeiten nach fehlgeschlagenen Osteosynthesen: Becken und Acetabulum. OPJournal 2004; 20: 202-208.

deutlich häufiger beim alten als beim jungen Patienten $[11,12,14]$.

\section{Diagnostik der Pseudarthrose am Sakrum}

In der Zeit, in der Beckenringfrakturen lediglich durch 3 konventionelle Röntgenbilder des hinteren Beckenrings (Becken a.-p., Inlet, Outlet) erkannt werden mussten, brauchte es schon einen hohen Kenntnisstand des Untersuchers, um Sakrumfrakturen direkt zu erkennen. Es wurden viele Hilfslinien, sekundäre Zeichen etc. genutzt, um Frakturen auf konventionellen Bildern zu detektieren. In Zeiten der überall verfügbaren CT-Diagnostik werden inzwischen nahezu alle Patienten mit vorderen Beckenringfrakturen einer Diagnostik auch des hinteren Beckenrings unterzogen und somit Frakturen im Sakrumbereich deutlich häufiger erkannt $[4,19]$.

Durch die zunehmende Zahl alter und ältester Patienten mit Frakturen des Beckenrings nach sog. Niedrigenergietrauma (z. B. Sturz vom Stuhl oder ein Sich-neben-denStuhl-Setzen ohne hohe Energieeinwirkung), die in der Notfallambulanz oder Sprechstunde vorgestellt werden, können aber auch mit Anwendung der CT diese sog. Insuffizienzfrakturen am Sakrum übersehen werden, da sie zum Untersuchungszeitpunkt nur als Fissuren vorhanden sind und somit gar nicht als „richtige“ Frakturen erkennbar werden $[15,18]$. Lediglich durch die konsequente Weiterführung der Diagnostik bei entsprechender Schmerzsymptomatik des Patienten mit einer Szintigrafie (eher historisch) oder der heute gebräuchlichen, hochauflösenden MRT des Beckens oder einer SPECT/CTUntersuchung (SPECT: Single Photon Emission Computed 

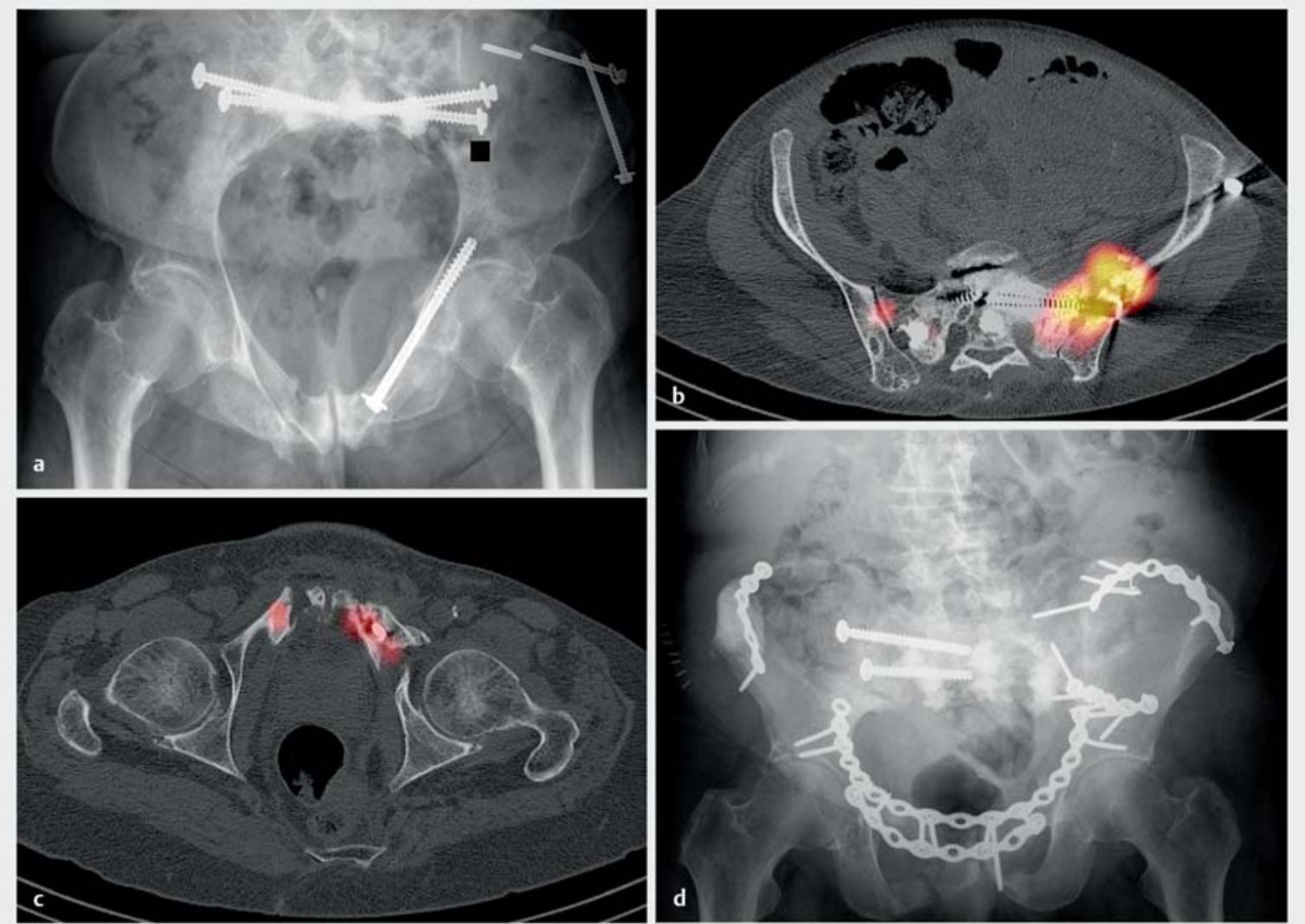

Abb. 3 Inlet-Aufnahme bei nicht verheilter transiliosakraler Luxationsfraktur mit nicht geheilten Schambeinastfrakturen beidseits mit posteriorer Translation und Innenrotation des Hemipelvis links bei Status nach Schraubenosteosynthesen am Beckenkamm und Schambeinast links und Status nach Sakroplastik (Zementierung des Sakrums) und S-I- und S-II-Schrauben beidseits nach Sakruminsuffizienzfrakturen (a). In einer SPECT/CT-Untersuchung (axiale Rekonstruktionen) zeigen sich die Aktivitätszonen als Ausdruck der Pseudarthrosen und Defektzonen am hinteren Beckenring (b) und am vorderen Beckenring (c). Beckenübersicht 2 Monate nach Außenrotation des Hemipelvis links, Doppelplattenosteosynthesen und Beckenspaninterposition am vorderen Beckenring und Plattenosteosynthese der Beckenschaufel über einen dorsalen Zugang (d) in einem 2-stufigen Verfahren. Quelle: Keel MJB, Bastian JD. Spätfolgen - Beckenchirurgie. OP-Journal 2018; 34: 48-52.

Tomography) kann eine Fraktur durch die Darstellung des ausgedehnten Frakturhämatoms im Sakrum zum Erkennen der Fraktur führen [12,18]. Ein Bone Bruise des Sakrums ist aber hierbei selbstverständlich nur bei entsprechender Anamnese und Klinik des Patienten als Frakturhämatom zu verstehen und nicht nur als „Prellung“ des Knochens.

Zusammenfassend sollte daher bei Beckenringfrakturen mit Beteiligung des Sakrums eine stufenweise Eskalation der Diagnostik erfolgen. Zunächst ist eine Beckenübersicht in Kombination mit einer Inlet-/Outlet-Aufnahme durchzuführen. Sind hier bereits Dislokationen kraniokaudal (Outlet) oder anterior-posterior (Inlet) zu erkennen, wird umgehend eine CT angeschlossen ( $\bullet \mathbf{A b b} \mathbf{3} \mathbf{3}$ ).

Ebenso bei Auftreten einer vorderen Beckenringfraktur mit einem horizontalen Frakturverlauf sollte eine CT zur Beurteilung des Sakrums angeschlossen werden. Wird keine Fraktur des hinteren Beckenrings oder des Sakrums gefunden, kann zunächst konservativ-funktionell behandelt und der Patient an Gehstützen ggf. mit einer Teilbelastung der betroffenen Seite mobilisiert werden. Klagt der Patient dann im Verlauf nach 3 Wochen trotz Schmerzmittelgabe über fortgesetzt tief lumbale Schmerzen oder nach einer initialen Phase der Schmerzabnahme wieder über zunehmende Schmerzen, sollte spätestens nach 4-6 Wochen eine MRT des Beckens für Klarheit bez. der Mitverletzung des Sakrums sorgen. Eine reine Steigerung der Schmerzmittelgabe im Verlauf und Abwarten ohne Fortführung der Diagnostik verzögert die Frakturerkennung und mindert die Chance, durch eine adäquate Therapie eine Ausheilung der Verletzung zu erreichen. Nur eine zielgerichtete und konsequente Weiterführung der Diagnostik verhindert somit letztlich die Entstehung einer Pseudarthrose im Sakrum. Die entsprechende klinische Symptomatik und der radiologische Nachweis der Sakrumfraktur sollten daher zu einer kon- 
sequenten Weiterbehandlung, ggf. auch frühzeitigen Operation, am Sakrum führen. Diese kann zu diesem Zeitpunkt der Behandlung durchaus immer noch minimalinvasiv erfolgen $[4,5,7,18,20-23]$.

\section{Behandlung der Sakrumfraktur}

Häufig werden Patienten mit Typ-B-Beckenringverletzungen bzw. ältere Patienten mit Insuffizienzfrakturen des Beckens (Typ C+D nach Rommens) konservativ behandelt und unter einer analgetischen Standardtherapie und mithilfe von Physiotherapie unter Einsatz verschiedener Hilfsmittel mobilisiert (z. B. mit einem Rollator oder Unterarmgehstützen) $[20,21]$. Eine notwendige Teilbelastung zur mechanischen Schonung der betroffenen Frakturseite können die alten Patienten häufig aber nicht richtig einhalten. In der Folge kann es durch die fortgesetzte Mikrobewegung in der Fraktur unter der Vollbelastung zu einer fortgesetzten Instabilität im Sakrumbereich kommen. Die Kallusbrücken, die sich zur Heilung bilden, werden immer wieder destruiert und dies führt letztlich mechanisch zu einer Frakturheilungsstörung im hinteren Beckenring, zunächst also zu einer verzögerten Frakturheilung am Sakrum $[10,7,14,18]$.

Zu diesem Zeitpunkt sollte die konservative Behandlung verlassen und eine minimalinvasive operative Behandlung der Sakrumfraktur erfolgen. Diese wird heute durch eine standardisierte, transiliosakrale Schraubenosteosynthese der Sakrumfraktur mit und ohne Stabilisierung des vorderen Beckenrings minimalinvasiv umgesetzt [1,6, 7 , $28,17,22-26]$. Auch perkutan eingeschobene ilioiliakale Plattenosteosynthesen oder perkutane Fixateur-interneMontage einseitig oder beidseitig kommen inzwischen regelhaft zur Anwendung. Kombiniert werden die o.a. Methoden mit einer Zementaugmentierung, die z. B. bei der Fixateur-interne-Stabilisierung zu einer höheren Schraubenfestigkeit beiträgt. Reine Zementauffüllungen des Sakrums als sog. Sakroplastie werden durchaus kontrovers diskutiert, da ein hohes Risiko der Zementleckage mit Nervenreizungen oder -ausfällen auftreten kann.

\section{Operative Therapie der Pseudarthrose am Sakrum}

Durch eine über Monate fortgesetzte Frakturheilungsstörung im Sakrum entsteht eine bindegewebige Pseudarthrose im ehemaligen Frakturverlauf. Besteht diese nach 6 Monaten fort, wird definitionsgemäß von einer Pseudarthrose am Sakrum gesprochen. Eine zu diesem Zeitpunkt durchgeführte, rein mechanisch stabilisierende Operation am Sakrum z. B. durch die o. a. minimalinvasive Schraubenosteosynthese (auch in zementaugmentierter Form) kann das Problem längerfristig allein nicht lösen $[2,12,26]$. Ebenso wie in anderen Regionen des Körpers muss bei einer bestehenden Pseudarthrose im Sakrum eine offen chirurgische Ausräumung der Pseudarthrose, eine Anlagerung von Spongiosa oder Fremdknochen und eine stabile Osteosynthese geplant und umgesetzt werden $[2,13,26]$.

Diese zumeist sehr ausgedehnte Operation stellt einen Revisionseingriff dar, und der Patient muss über die möglichen Komplikationen ausführlich aufgeklärt werden. Blutverlust, Nachblutungen, Nervenverletzungen und Nekrosen von Haut und Muskulatur über dem dorsalen Sakrum sind dabei spezifische Komplikationen, die nicht unerwähnt bleiben sollten.

\section{Durchführung der Operation}

Die Planung der Operation umfasst die Bereitstellung von ausreichend Blutkonserven (2-4 Erythrozytenkonzentrate), möglichst zusätzlich die Verwendung eines Cell Savers und eine postoperativ adäquate Nachbetreuung des Patienten auf einer chirurgischen Intensivstation, ggf. unter Verwendung spezieller Lagerungstechniken wie Luftstrommatratze oder Freilagerung des Sakrumbereichs, um sekundäre Haut- und Weichteilnekrosen durch Druck zu verhindern. Auch die Mobilisierung des operierten Patienten und hierfür notwendige Rehakonzepte bei vorbestehender Immobilität des Patienten sind Teil der Vorbereitung auf die Operation gemeinsam mit dem Patienten. Durch postoperativ enge Patientenführung gemeinsam mit dem weiterbehandelnden Arzt/Facharzt und durch eigene Nachkontrolle des Ergebnisses nach 3, 6, 12 und 24 Monaten sollte bereits im Vorfeld mit dem Patienten und seinem direkten Behandler auch die Nachhandlung besprochen werden. Bereits bei der präoperativen Vorbereitung kann der Patient durch Mitgabe und Nutzung eines Triflow-Geräts zum Atemtraining, durch Optimierung z. B. einer Insulintherapie bei bestehendem Diabetes mellitus, durch Verbesserung der kardialen Ausgangssituation oder präoperativer Steigerung des $\mathrm{Hb}$ Wertes optimal auf den großen Eingriff eingestellt werden. Ebenso kann sich auch der Patient selbst durch eine Verbesserung seines Ernährungszustandes auf die anstehende Operation vorbereiten. Es handelt sich letztlich um eine elektiv durchzuführende, notwendige Operation, zu der der Patient und der Chirurg über einen längeren Zeitraum ausreichend vorbereitet sein sollten. Der Patient sollte durch eine eigene eingehende Untersuchung, durch ausführliche Aufklärung und vorliegende vollständige Diagnostik durch den Operateur zur OP vorbereitet werden.

Ziel der Operation am pseudarthrotischen Sakrum ist dabei einerseits die mechanische Stabilisierung des Sakrums durch eine ausreichend dimensionierte und stabile Osteosynthese und andererseits die Herstellung ausreichend biologisch aktiven Knoches durch Ausräumung des Bindegewebes und Anlagerung von Spongiosa, um eine sichere Ausheilung zu erzielen.

Am Tag vor der Operation sollte der Patient abgeführt haben, um eine direkt postoperativ häufig zu beobach- 
tende Obstipation zu verhindern. Eine perioperative Antibiotikatherapie ist bei diesem Eingriff indiziert, es sollte daher ca. eine halbe Stunde vor dem Eingriff ein Cephalosporin der 2. Generation durch die Anästhesie appliziert werden. Ebenso muss auf eine ausreichende postoperative Schmerzmedikation mit Opioiden und/oder einer zusätzlichen patientengesteuerten Schmerztherapie geachtet werden, ggf. sollte auch eine peridurale Medikamentenapplikation zur Schmerztherapie durchgeführt werden. Dies insbesondere auch unter dem Hintergrund, dass es sich häufig um chronische Schmerzpatienten aufgrund des zeitlichen Verlaufs bei einer Pseudarthrose handelt.

Eine medikamentöse Prophylaxe von heterotopen Ossifikationen durch ein verbleibendes Hämatom im muskulären Bereich des Zugangs sollte bei fehlenden Kontraindikationen ebenso berücksichtigt werden.

\section{Lagerung und OP-Technik}

Der Patient wird in Bauchlage auf einem röntgendurchlässigen Carbontisch gelagert. Auf ein ausreichendes Wärmemanagement während der OP sollte zur Verhinderung einer Auskühlung und konsekutiven Gerinnungsstörung unbedingt geachtet werden.

Nach sterilem Abwaschen und Abdecken wird eine mediane oder mediolaterale gerade Längsinzision bei zentralen/transforaminalen Pseudarthroseverläufen durchgeführt. Bei Pseudarthrosen im Bereich der Ala sacralis ist eine direkt über der Pseudarthrose gelegene Längsinzision im Bereich der dorsalen Iliumkämme sinnvoll, die nach kranial Richtung LWS oder auch nach kaudal Richtung Sakrumspitze verlängert werden kann.

Nach Hautinzision und Durchtrennung der Subkutis wird die Muskelfaszie der autochthonen Sakrummuskulatur dargestellt. Durch eine V-förmige Inzision der Muskelfaszie zwischen den Dornfortsätzen des Sakrums auf der einen Seite und dem Ilium auf der anderen Seite wird ein kranial gestielter Muskellappen geschaffen, der nach kopfwärts präpariert und nach Freilegung des Sakrums umgeschlagen werden kann. Es muss peinlich darauf geachtet werden, dass dieser Muskellappen während der gesamten OP feucht gehalten wird, um eine rasch eintretende Schrumpfung des Muskels und eine Nekrose zu verhindern.

In der Regel reicht ein einseitiges Vorgehen aus, bei zentralen Pseudarthrosen muss bilateral präpariert werden. Laterale Begrenzung ist die dorsale Spina iliaca posterior superior und die dorsale Crista des Iliums bzw. die Sakroiliakalfuge. Nach kranial sollte der obere Rand des S-I-Pedikels ertastet werden können. Kaudal ist der knöcherne Rand des Sakrums begrenzend bzw. nach distal der Beginn des Os coccygis. Eine komplette Abtrennung des Lig. sacrospinale oder des Lig. sacrotuberale ist nicht zwingend notwendig. Diese Beckenbodenbänder müssen dann abgelöst werden, wenn eine zusätzliche Reposition zur Kompression der ausgeräumten Pseudarthrose notwendig ist. Nur durch die Ablösung der Beckenbodenbänder kann eine ausreichende Bewegungsfreiheit der Fragmente zur Reposition erzielt werden. Selbstverständlich muss bei der Präparation auf die Neuroforamina geachtet werden und auch bei der Ausräumung der Pseudarthrose muss insbesondere auf die nach ventral ziehenden Nerven geachtet werden. Eventuell ist die operative Darstellung der Nervenabgänge unter Nutzung eines Operationsmikroskops oder einer Lupenbrille zielführend.

Anschließend wird die Pseudarthrose dargestellt und die bindegewebigen Anteile ausgeräumt, bis sich frischer spongiöser Knochen zeigt. Ist ein entsprechender Spalt entstanden, wird dieser mit Eigenspongiosa aus dem Beckenkamm aufgefüllt bzw. bei einem kleinen Spalt (bis $5 \mathrm{~mm}$ ) adaptierend mit Repositionszangen direkt reponiert und Spongiosa aus dem Beckenkamm angelagert. Die Fragmente werden anschließend mit 2 winkelstabilen 3,5-mm-Kleinfragmentplatten oder 2,4-mm-Platten in Höhe des S-I- und des S-II-Pedikels quer stabilisiert. Ebenso kann durch jeweils eine transiliosakrale Stellschraube in Höhe S I und/oder S II die Kompression der Fraktur erreicht werden. Eine Kombination der beiden Methoden führt zu einer Stabilität in 2 Ebenen und damit zu einer höheren Sicherung der Pseudarthrose. Grundsätzlich sollten Implantate in 2 Höhen des Sakrums verwendet werden, um einerseits die Kompression der ausgeräumten Pseudarthrose auf der ganzen sakralen Länge und andererseits eine Rotationssicherung der Fragmente selbst zu gewährleisten.

Nach Abschluss der Stabilisierung wird der gebildete Muskellappen wieder zurückgeklappt und distal mit einer Naht fixiert. Anschließend Verschluss der Faszie auf beiden Schenkeln des V-förmigen Zugangs. Danach Wundverschluss durch Einzelknopfsubkutannähte und Hautnaht. Ob eine Redon-Drainage zum Abschluss der OP eingelegt wird, ist abhängig von der erreichten Bluttrockenheit. Gegen eine Drainage spricht die Nähe zum Anus und des damit deutlich erhöhten Risikos einer sekundären Infektion der Wunde, für eine Drainage die zu erzielende Bluttrockenheit im Muskelbett. Wenn sie notwendig erscheint, sollte sie nach kranial ausgeleitet werden.

\section{Nachbehandlung}

In der direkt postoperativen Zeit sollte eine ausreichende Analgesie und eine antiphlogistische Therapie zur Ossifikationsprophylaxe verordnet werden. Außerdem muss die Wunde unbedingt trocken gehalten werden, um eine sekundäre Infektion zu vermeiden. Gegebenenfalls sollte eine Luftstrommatratze zur Lagerung eingesetzt werden, wenn der Patient postoperativ nicht direkt mobilisierbar ist. Physiotherapeutisch sollte der Patient mit einem Geh- 
bock zur Gewichtsentlastung oder direkt mit Unterarmgehstützen mobilisiert werden. Eine Teilbelastung macht nur bei verständigen und mitarbeitenden Patienten mit Fähigkeit der Umsetzung und bei einseitigen lateral gelegenen Pseudarthrosen Sinn. Zentrale Pseudarthrosenverläufe müssen eine Vollbelastung aushalten, da auch die Mobilisation des Patienten mit Rollstuhl eine direkte Belastung der Fragmentzone darstellen würde.

\section{Komplikationen}

Nachblutungen im Wundgebiet und eine fortgesetzte Sekretion sollten unbedingt vermieden werden. Gegebenenfalls ist eine frühzeitige oder sogar eine fest eingeplante Revisionsoperation mit erneutem Wunddébridement und einer Jet-Lavage sowie dann erneutem Wiederverschluss der Wunde sinnvoll. Dies sollte bereits präoperativ mit dem Patienten besprochen werden, dass dies durchaus ein Teil des normalen Therapieverlaufs sein kann. Eine fortgesetzte Antibiotikatherapie ist weder erforderlich noch sinnvoll.

Durch die tägliche Wundpflege, die häufige Umlagerung des Patienten auf die Seite oder Lagerung auf einer geeigneten Luftstrommatratze muss unbedingt die Nekrosebildung am Muskellappen und die damit häufig einhergehende Infektion verhindert werden. Die Sauberkeit beim Toilettengang ist dem Patienten bereits vor dem Eingriff als zwingende Pflicht aufzuerlegen.

\section{Fazit für die Praxis}

Grundsätzlich sind Sakrumpseudarthrosen selten. Radiologisch scheinbar eindeutige Pseudarthrosen am Sakrum bei klinisch asymptomatischen Patienten sind dabei ebenso möglich wie klinisch stark schmerzhafte Patienten, die bei adäquater Umsetzung der Behandlungsmaßnahmen keine Pseudarthrose entwickeln. Primäres Übersehen einer Sakrumfraktur kann ebenso durch die fortgesetzt mechanische Instabilität wie auch bei den älteren Patienten eine Insuffizienzfraktur am hinteren Beckenring zur Entstehung einer Pseudarthrose beitragen. Konsequente Weiterführung der Diagnostik und frühzeitige minimalinvasive Stabilisierung, insbesondere von Insuffizienzfrakturen des Beckenrings beim alten Patienten, können eine Entstehung einer Pseudarthrose im Sakrum suffizient verhindern.

Pseudarthrosenchirurgie ist Komplikationsmanagement! Ebenso wie im Bereich anderer Körperregionen, die sicher häufiger von einer Pseudarthrose betroffen sind, sind die Herstellung der mechanischen Belastbarkeit des hinteren Beckenrings und die Schaffung der biologischen Voraussetzungen zur knöchernen Heilung unter Verhinderung einer Infektionsentstehung die wichtigsten Maßnahmen zur Behandlung einer Pseudarthrose am Sakrum.
Interessenkonflikt

Die Autoren geben an, dass kein Interessenkonflikt besteht.

Autorinnen/Autoren

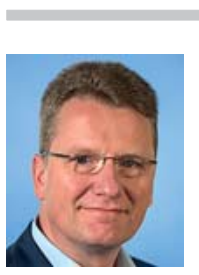

\section{Ulf Culemann}

Prof. Dr. med., Chefarzt der Klinik für Unfallchirurgie, Orthopädie und Neurotraumatologie, Allgemeines Krankenhaus Celle

Korrespondenzadresse

\section{Ulf Culemann}

Klinik für Unfallchirurgie, Orthopädie und Neurotraumatologie Allgemeines Krankenhaus Celle

Siemensplatz 4

29221 Celle

Tel.: 0 5141/721101

Fax: 05141/721109

ulf.culemann@akh-celle.de

\section{Literatur}

[1] Oberkircher L, Ruchholtz S, Rommens PM et al. Osteoporotic pelvic fractures. Dtsch Arztebl Int 2018; 115: 70-80. doi:10.3238/arztebl.2018.0070

[2] Mears DC, Velyvis J. Surgical reconstruction of late pelvic posttraumatic nonunion and malalignment. J Bone Joint Surg Br 2003; 85: 21-30

[3] Culemann U, Reilmann H. Verletzungen des Beckenringes, Unfallchirurg 1997; 100: 487-496

[4] Pohlemann T, Gänsslen A, Tscherne H. Die Sakrumfraktur. Unfallchirurg 2000; 103: 769-786

[5] Culemann U, Tosounidis G, Reilmann H, Pohlemann T. Pelvic fracture. Diagnostics and current treatment options. Chirurg 2003; 74: 687-698

[6] Pohlemann T, Tscherne H, Baumgärtel F et al. Beckenverletzungen: Epidemiologie, Therapie und Langzeitverlauf. Unfallchirurg 1996; 99: 160-167

[7] Tscherne H, Pohlemann T, Hrsg. Becken und Acetabulum. Berlin, Heidelberg, New York: Springer; 1998

[8] Denis F, Steven D, Comfort T. Sacral fractures: an important problem. Retrospective analysis of 236 cases. Clin Orthop Relat Res 1988; 227: 67-81

[9] Nothofer W, Thonke N, Neugebauer R. Die Therapie instabiler Sakrumfrakturen bei Beckenringbrüchen mit dorsaler Sakrumdistanzosteosynthese. Unfallchirurg 2004; 107: 118-128

[10] Matta JM, Dickson KF, Markovich GD. Surgical treatment of pelvic nonunions and malunions. Clin Orthop Relat Res 1996; (329): 199-206

[11] Gautier E, Rommens PM, Matta JM. Late reconstruction after pelvic ring injuries. Injury 1996; 27 (Suppl. 2): B39-B46

[12] Kanakaris NK, Angoules AG, Nikolaou VS et al. Treatment and outcomes of pelvic malunions and nonunions: a systematic review. Clin Orthop Relat Res 2009; 467: 2112-2124

[13] Mears DC. Management of pelvic pseudarthroses and pelvic malunion. Orthopade 1996; 25: 441-448 
[14] Oransky M, Tortora M. Nonunions and malunions after pelvic fractures: why they occur and what can be done?. Injury 2007; 38: 489-496

[15] Taller S, Srám J, Lukás R et al. Nonunions or malunions of pelvic fractures. Acta Chir Orthop Traumatol Cech 2009; 76: 121127

[16] Vanderschot P, Daenens K, Broos P. Surgical treatment of posttraumatic pelvic deformities. Injury 1998; 29: 19-22

[17] Chip Routt ML jr., Simonian PT. Closed reduction and percutaneous skeletal fixation of sacral fractures. Clin Orthop Relat Res 1996; (329): 121-128

[18] Rommens PM, Hoffmann A. Comprehensive classification of fragility fractures of the pelvic ring: recommendations for surgical treatment. Injury 2013; 44: 1733-1744

[19] Jackson H, Kam J, Harris J. The sacral arcuate lines in upper sacral fractures. Radiology 1982; 145: 35-39

[20] Maier GS, Kolbow K, Lazovic D et al. Risk factors for pelvic insufficiency fractures and outcome after conservative therapy. Arch Gerontol Geriatr 2016; 67: 80-85

[21] Tosounidis G, Wirbel R, Culemann U et al. [Misinterpretation of anterior pelvic ring fractures in the elderly]. Unfallchirurg 2006; 109: 678-680

[22] Josten C, Höch A. [Fractures of the sacrum: operative/conservative. Pro: why insufficiency fractures of the sacrum should be treated operatively]. Die Wirbelsäule 2017; 1: 31-40
[23] Rollmann MF, Herath SC, Kirchhoff F et al. Pelvic ring fractures in the elderly now and then-a pelvic registry study. Arch Gerontol Geriatr 2017; 71: 83-88

[24] Schildhauer TA, Bellabarba C, Nork SE et al. Decompression and lumbopelvic fixation for sacral fracture-dislocations with spino-pelvic dissociation. J Orthop Trauma 2006; 20: 447-457

[25] Gänsslen A, Pape HC, Lehmann U et al. Die operative Therapie von instabilen Sacrumfrakturen. Zentralbl Chir 2003; 128: 4045

[26] Keel MJB, Bastian JD. Spätfolgen - Beckenchirurgie. OP-Journal 2018; 34: 48-52

[27] Helfet D. Alpha-numeric classifications for pelvic fractures. In: Surgery of the Pelvis and Acetabulum: an international Consensus. Conference Proceeding. Pittsburgh: 1992

[28] Isler B, Ganz R. Klassifikation der Beckenringverletzung. Unfallchirurg 1990; 93: 289-302

\section{Bibliografie}

DOI https://doi.org/10.1055/a-0903-3201

OP-JOURNAL 2019; 35: 301-308 @ Georg Thieme Verlag KC Stuttgart · New York ISSN 0178-1715 